\title{
Implication of Platelet Rich Plasma- As an Adjuant to Hydroxyapatite in the Treatment of Periodontal Infra Bony Defects -A Controlled Randamized Clinical and Radiological Study
}

\author{
Dr Tazeen Dawood \\ Assistant Professor, Department Of Periodontics, College Of Dentistry, Jazan University,
} Saudi Arabia.

\begin{abstract}
Platelet-rich plasma (PRP) contains high concentration of polypeptide growth factors that enhances periodontal regeneration when combined with graft materials. The aim of the present study was to evaluate the regenerative potential of a biogradable ceramic porous hydroxyapaptite and its combination with platelet rich plasma in the treatment of human periodontal intrabony defects. 20 periodontal interproximal intra-bony defects in 10 systemically healthy patients diagnosed with chronic periodontitis were included in the study. The defects were randomly divided into two group A included the defects treated by bone graft alone and, group B a combination of platelet rich plasma and hydroxyapatite. The clinical and radiographic parameters assessed were plaque index, gingival index, probing pocket depth, relative attachment level, at baseline 3 months and 6 months. At the end of 6 months both groups showed significant changes in clinical parameters, the test showed better clinical outcomes when compared to control group, the probing depth reduction of group A was $9.8 \pm 2.62$ to $4.98 \pm 0.58 \mathrm{~mm}$, versus $10.58 \pm 4.5 \mathrm{~mm}$ to $3.5 \pm 0.96 \mathrm{~mm}$ at the end of 6 months for group B. The GroupA showed (RAL) of $11.08 \pm 1.49 \mathrm{~mm}$ to $5.3 \pm 0.67 \mathrm{~mm}$ versus, $12.5 \pm 1.67 \mathrm{~mm}$ to $4.4 \pm 2.4 \mathrm{~mm}$ at the end of 6 months for group B, radiographic defect fill values:, Group A showed $1.65 \pm 0.30$ $\mathrm{mm}$, GroupB showed $3.98 \pm 2.6 \mathrm{~mm}$.
\end{abstract}

Conclusion: combination of Platelet rich plasma with bone graft showed significantly better treatment outcome compared to bone graft alone..

Keywords: clinical studies, periodontal regeneration, platelet rich plasma tissue engineering

\section{Introduction}

Periodontitis is defined as an inflammatory disease of the supporting tissues of the teeth, resulting in the progressive destruction of the periodontal ligament and alveolar bone.[1 ]The ultimate goal of periodontal therapy is to protect and maintain the patients natural dentition for optimal comfort, function and esthetic appearance and to restore as much of lost tooth structure as possible. Periodontal regeneration has reached new horizons with the advent of new diagnostic aids, new biomaterials and new surgical procedures which are capable of improving the periodontal surgery outcomes in terms of percentages of success. Platelet rich plasma enriched with growth factors is considered to be a "new frontier" and a new innovation in periodontics aiming for periodontal regeneration, it is widely used in various surgical fields including head and neck surgery, otolaryngology, cardiovascular surgery, and maxillofacial surgery .The implications of PRP in dentistry include, sinus lift procedures, onlay grafts, particulate grafts, alveolar cleft palate repair, oral/nasal fistula repair, post-operative hemostasis of bone graft donor sites, continuity defects of the mandible and hemophiliacs undergoing surgery.[2,3] Porous hydroxyapatite acts as a scaffold for the in growth of connective tissue and osteogenic cells from the existing bone surfaces into adjacent bone graft which get transformed into osteocytes in the defect site. it is biocompatible, non-toxic, resorbable, and non-inflammatory; cause no immunological, foreign-body, or irritating response; the combination of PRP and a hydroxyapatite [HA] osseous graft has been demonstrated to be efficacious in the treatment of periodontal endosseous defects. . However, only a few clinical trials have tested the efficacy of a combination of PRP and bone grafts in the treatment of intrabony defects $[4,5,6]$. Therefore, the purpose of this controlled, randomized comparative clinical and radiographic study was to determine the regenerative potential of a combination of HA and PRP with HA alone in treatment of periodontal intrabony defects.

\section{Materials and methods}

The protocol of this study was approved by the Ethical Committee of Vinayaka Missions University, This randomized clinical control trial was carried out in the Department of Periodontics, Vinayaka Misssions Sankarachariyar Dental College and Hospital, Salem.. The study sample included 20 periodontal infrabony defects in 10 patients, 5 males and 5 females, aged between $25-45$ years. The defects were randomly divided 
into two groups and followed up for a period of 3 and 6 months .Group A: bone graft (Experimental). Group B: PRP + Bone graft (Experimental) .Patients without any history of systemic disease or compromising medical conditions having a clinical evidence of periodontal pocket of more than $5 \mathrm{~mm}$ and radiographic evidence of intra bony defect. were included in the study. Patients having unacceptable oral hygiene maintaince after phase 1 , history of antibiotics or other medications affecting the periodontium, within the previous 6 months, pregnant women, lactating mothers and smokers were excluded from the study. The protocol of the study was explained to the patient and an consent was taken. Phase 1 therapy included thorough full mouth scaling and root planing. Six weeks following phase-1 therapy, a periodontal reevaluation was performed to confirm the suitability of the sites for the study.

The following clinical parameters were assessed at baseline, three \& six months post surgery. Plaque index(PI) (Sillness and Loe), Gingival index(GI) ( Loe and Sillness) Probing pocket depth(PPD)relative attachment level, which were recorded using a Williams graduated periodontal probe, a customized acrylicstent with a guiding groove was made to standarlize the direction of probing pre and post surgery . Radiographic assessment was done by digital substraction radiography and the results were analysed by the help of image analysis software (Motic-china in corporation ltd) to see changes between preoperative and post operative radiographs. Bone graft material used was a resorbable porous hydroxyapatitie( biograft ) of particle size 0.25 $1 \mathrm{~mm}$. The surgical procedure and the method of preparation of platelet rich plasma was done according to the procedure described by Kazuhiro Okuda et al.(2005)[7]. Once the recipient defect site was prepared , PRP was activated by clot initiator $10 \%$ calcium chloride to attain a white sticky buffy coat which was mixed with porous hydroxyapatite (biograft) and applied to the surgical defects. Flaps were repositioned to the pre-surgical level to achieve primary closure. Systemic administration of Amoxicillin 500mg thrice daily for 5 days and a NSAID thrice daily for 5 days and $0.2 \%$ chlorhexidine gluconate rinse twice daily for a period of 2 weeks was prescribed to the patients. Sutures were removed one week post surgery and oral hygiene instructions were reinforced.The clinical parameters were recorded at three and six months and radiographic recording was done after 6 months postoperatively .

\section{Statistical Analysis}

The data at baseline, 3 months and 6 months after surgery was subjected to statistical analysis using:ANOVA-Paired t test. All the data was expressed as mean (SD). Statistical analysis was performed using a commercial SPSS version II. One way analysis of variance was applied to examine the difference among the two groups. Clinical and radiographic parameters were subjected to student t test and the "t" and " $p$ " values were obtained with appropriate levels of significance.

\section{Results}

Each subject participating in the study demonstrated excellent oral hygiene and a generally healthy gingival condition throughout the study. GROUP A showed a mean plaque index of $2.96 \pm 0.76$ at Baseline, $1.50 \pm 0.42$ at three months and $1.32 \pm 0.33$ at the end of six months, whereas group B showed a mean plaque index of $2.37 \pm 0.97$ at baseline, $1.49 \pm 0.22$ at three months and was reduced to $1.12 \pm 0.32$ at the end of 6 months, thus the results were statistically significant. $(\mathrm{p}<0.337)$ "Table" 1 Gingival index- Group A showed a mean gingival index of $2.24 \pm 1.10$ at Baseline, reduced to $0.83 \pm 0.2$ at three months and further reduced to $0.45 \pm 0.15$ at the end of six months. Group B showed statistically significant gingival index values $1.75 \pm 0.51$ at baseline, $0.78 \pm 0.25$ at three months and $0.6 \pm 0.12$ at the end of 6 months, $(p<0.001)$. oral hygiene instructions were reinforced.

"Table 2". Group A showed a mean probing pocket depth (PPD) of $9.8 \pm 2.62 \mathrm{~mm}$ at Baseline, $6.5 \pm 1.83$ $\mathrm{mm}$ at three months and $4.98 \pm 0.58 \mathrm{~mm}$ at the end of six months. The mean probing pocket depth in the GroupB was $10.58 \pm 4.5 \mathrm{~mm}$ at baseline, $5.60 \pm 2.4 \mathrm{~mm}$ at three months and $3.5 \pm 0.96 \mathrm{~mm}$ at the end of 6 months, results were statistically significant. $(\mathrm{p}<0.001)$."Table 3". GroupA showed a mean relative attachment level (RAL) of $11.08 \pm 1.49 \mathrm{~mm}$ at Baseline, $7.1 \pm 2.36 \mathrm{~mm}$ at 3 months and $5.3 \pm 0.67 \mathrm{~mm}$ at the end of six months .and Group B $12.5 \pm 1.67 \mathrm{~mm}$ at baseline, $7.84 \pm 3.83 \mathrm{~mm}$ at three months and $4.4 \pm 2.4 \mathrm{~mm}$ at the end of 6 months, which were statistically significant. ( $<<0.001$ "Table4".The radiographic defect depth values at baseline: Group A showed $3.23 \pm 1.10 \mathrm{~mm}$, and Group B showed $5.85 \pm 3.04 \mathrm{~mm}$. $\quad \mathrm{P}=0.120$ "Table 5". The radiographic defect depth values at six months: Group A showed $2.28 \pm 0.76 \mathrm{~mm}$, and Group B showed $2.24 \pm 1.32 \mathrm{~mm} \mathrm{P=}$ 0.706. "Table 6".The radiographic defect fill values: Group A showed $1.65 \pm 0.30 \mathrm{~mm}$, Group B showed 3.98 \pm 2.6 mm. $\mathrm{p}=0.40$ "Table 7 ". 
Table 1: Mean changes in the plaque index within and across the two study groups

\begin{tabular}{|l|l|l|l|l|l|}
\hline \multirow{2}{*}{ PI } & GROUP A & \multicolumn{3}{|l|}{ GROUP B } & \multirow{2}{*}{ P value } \\
\cline { 2 - 5 } & Mean & SD & Mean & SD & \\
\hline Baseline & 2.96 & \pm 0.76 & 2.37 & \pm 0.97 & 0.371 \\
\hline 3 months & 1.50 & \pm 0.42 & 1.49 & \pm 0.22 & 0.174 \\
\hline 6 months & 1.32 & \pm 0.26 & 1.12 & \pm 0.32 & 0.534 \\
\hline P value & $<0.001$ & $<0.337$ & - \\
\hline
\end{tabular}

Table 2: Mean changes in the gingival index within and across the study groups

\begin{tabular}{|l|l|l|l|l|l|}
\hline \multirow{2}{*}{ GI } & \multicolumn{3}{|l|}{ GROUP A } & GROUP B & \multirow{2}{*}{ P value } \\
\cline { 2 - 5 } & Mean & SD & Mean & SD & \\
\hline Baseline & 2.24 & \pm 1.10 & 1.75 & \pm 0.51 & 0.621 \\
\hline 3 months & 0.83 & \pm 0.23 & 0.78 & \pm 0.25 & 0.025 \\
\hline 6 months & 0.45 & \pm 0.15 & 0.6 & \pm 0.12 & 0.26 \\
\hline Pvalue & $<0.001^{* *}$ & $<0.001^{* *}$ & - \\
\hline
\end{tabular}

Table 3: Mean changes in the probing pocket depth within and across the study groups.

\begin{tabular}{|l|l|l|l|l|l|}
\hline \multirow{2}{*}{ PPD } & GROUP A & \multicolumn{3}{l|}{ GROUP B } & \multirow{2}{*}{ P value } \\
\cline { 2 - 5 } & Mean & SD & Mean & SD & \\
\hline Baseline & 9.8 & \pm 2.62 & 10.58 & \pm 4.5 & 0.754 \\
\hline 3 months & 6.5 & \pm 1.83 & 5.60 & \pm 2.4 & 0.595 \\
\hline 6 months & 4.98 & \pm 0.58 & 3.50 & \pm 0.96 & 0.107 \\
\hline P value & $<0.004^{* *}$ & $<0.021^{* *}$ & - \\
\hline
\end{tabular}

Table 5: Mean changes in the relative attachment level within and across the study groups

\begin{tabular}{|l|l|l|l|l|l|}
\hline \multirow{2}{*}{ CAL } & \multicolumn{4}{|l|}{ GROUP A } & \multicolumn{2}{l|}{ GROUP B } & \multirow{2}{*}{ P value } \\
\cline { 2 - 5 } & Mean & SD & Mean & SD & \\
\hline Baselin-e & 11.08 & \pm 1.49 & 12.5 & \pm 1.67 & 0.016 \\
\hline 3 months & 7.1 & \pm 2.36 & 7.84 & \pm 3.83 & 0.100 \\
\hline 6 months & 5.3 & \pm 0.67 & 4.4 & \pm 2.4 & $<0.001$ \\
\hline P value & $<0.001^{* *}$ & $<0.001^{* *}$ & - \\
\hline
\end{tabular}

Table 6: Mean changes in the radiographic defect depth at baseline and and six months across the study

\begin{tabular}{|l|l|l|l|l|l|}
\hline \multicolumn{2}{|c|}{ groups. } & \multicolumn{2}{l|}{ GROUP B } & P value \\
\cline { 2 - 5 } & Mean & SD & Mean & SD & \\
\hline $\begin{array}{l}\text { Radiographic defect } \\
\text { depth at baseline } \\
(\mathrm{mm})\end{array}$ & 3.23 & \pm 1.101 & 5.85 & \pm 3.04 & 0.120 \\
\hline $\begin{array}{l}\text { Radiographic defect } \\
\text { depth at six months } \\
(\mathrm{mm})\end{array}$ & 2.28 & \pm 0.76 & 2.24 & \pm 1.32 & 0.706 \\
\hline
\end{tabular}

Table 8: Mean changes in the radiographic defect fill at six months across the study groups.

\begin{tabular}{|c|c|c|c|c|c|}
\hline & \multicolumn{2}{|c|}{ GROUP A } & \multicolumn{2}{|c|}{ GROUP B } & \multirow{2}{*}{$P$ value } \\
\hline & Mean & SD & Mean & SD & \\
\hline $\begin{array}{l}\text { Radiographic defect } \\
\text { fill at six months } \\
(\mathrm{mm})\end{array}$ & 1.65 & \pm 0.30 & 3.98 & \pm 2.6 & $<0.004$ \\
\hline
\end{tabular}

\section{Discussion}

Guided bone regeneration is an important treatment modality which aims to increase the quality and quantity of host bone in alveolar defects. Today s practioners are available with a wide variety of regenerative materials including autografts, allogfafts and xenografts, platelet rich plasma, platelet rich fibrin, enamel matrix derivatives, pepgen 15 and stem cell therapy. Platelet rich plasma is a promising innovation in dentistry which utilizes the principles of tissue engineering to regenerative the periodontium lost by periodontal disease. It is defined as the volume of autologous plasma that has a platelet concentration alone as baseline. [8], the Components of platelet rich plasma include, platelet derived growth actor ,transforming - growth factor ,epidermal growth factor (E.G.F.),fibroblast growth factor ,Insulin like growth factor and platelet derived angiogenesis factor ,.high concentration of leukocytes (neutrophils, eosinophils), wound macrophage, and other phagocytic cells for biological debridement, histamines, serotonin, Adenosine diphosphate thromboxane. Growth factors are chief biologic modifiers and polypeptide hormones that stimulate a wide variety of cellular events including chemotaxis, proliferation, differentiation and production of extra cellular matrix proteins. Platelet derived growth factor was first discovered by Lynch\& Co-workers to promote regeneration of bone, cementum periodontal ligament. in late 1805 [9]. Purified platelet derived growth factor -BB when mixed with 
bone allograft results in periodontal regeneration in both class II furctions and interproximal intrabony defects, The Transforming growth factor-b initially activates fibroblasts and preosteoblasts to mitose and increase their numbers, and to mature to functioning osteoblasts. Continued Transforming growth factor-b secretion influences the osteoblasts to lay down bone matrix and the fibroblast to lay down collagen matrix to support capillary ingrowth.[10,11] Platelet rich plasma works by Increasing the tissue vascularity through increased angiogenesis of capillary budding in the graft by inducing endothelial cell mitosis, it acts as a chemoattractant for monocytes, macrophages and fibroblasts, it enhances collagen synthesis, increases the rate of epithelial and granulation tissue production, enhances osteogenesis. The high concentration of leukocytes in the buffy coat act as an antimicrobial. The present study was designed to evaluate platelet rich plasma as an adjuant to hydroxyapatite in treating intra-bony osseous defects.

Results from this investigation showed that a combination of platelet rich plasma + hydroxyapatite was effective in significantly improving the clinical and radiographic parameters between baseline and six months . The platelet rich plasma + hydroxyapatite group showed a significantly greater probing depth reduction of $(6.1 \pm 3.6 \mathrm{~mm})$ and relative attachment gain $(7.1 \pm 4.1 \mathrm{~mm})$ when compared to the saline hydroxyapatite who showed a probing depth reduction $(4.9 \pm 2.04 \mathrm{~mm})$, relative attachment level $(6.1 \pm 2.28$ $\mathrm{mm}$ ) Our clinical results were more favourable than the earlier investigations done by Kazuhiro Okuda et al 2005, the results of the present study were contradictory to the results found by Ferenc Dori et al 2007 [12] the results of group A where in accordance with Drizhal et al., (2010) [13] and Sukumar et al., (2011) [The efficacy of a combination of PRP and Hydroxyapatite in reduction of alveolar defect depth corroborate with the findings of Hanna Ret.al 2004, [14] Yilmaz S et.al 2011[15], Saini N et al 2011[16], Kaushick BT et.al 2011[17].Shreya Shetty et.al(2013) [18].Shakshi et.al have conducted a clinical study on platelet rich plasma and allograft and have concluded that platelet rich plasma enchances better regeneration with combined to allografts

\section{Conclusion}

There was a significant improvement in clinical parameters and radiographic dimensions within both the groups six months post surgery .A highly statistically significant difference in the percentage defect resolution was observed in sites treated with a combination of PRP and hydroxyapatite, thus PRP has proved to be more beneficial and an effective adjuant to bonegraft in the treatment of periodontal osseous defects.

\section{Limitations of the present study}

Further histological studies needs to be done to evaluate the outcome of platelet rich plasma. The long term effects of these treatment options need to be assessed with larger sample size and longer study period. In future, third generation probes could be used to overcome the problems with reliability of measurement of clinical recordings.

\section{References}

[1]. Carranza F.A. 1996: "Reconstructive osseous surgery". In Carranza FA Jr., Newman MG, Clinical Periodontology, $8^{\text {th }}$ Ed. W.B. Saunders; 622-639

[2]. Marx RE. Platelet rich plasma (PRP): What is PRP and what is not PRP? J Implant Dent. 2001;10(4):225-228.

[3]. Marx RE. Platelet rich plasma: evidence to support its use. J Oral Maxillofac Surg. 2004;62:489-496.

[4]. Lekovic V, Camargo PM, Weinlaender M, Vasilic N, Aleksic Z and Kenney EB. Effectiveness of a combination of platelet-rich plasma, bovine porous bone mineral and guided tissue regeneration in the treatment of mandibular grade II molar furcations in humans. J Clin Periodontol. 2003; 30:746-51

[5]. Camargo PM, Lekovic V, Weinlaender M, Vasilic N, Madzarevic M and Kenney EB. Platelet-rich plasma and bovine porous bone mineral combined with guided tissue regeneration in the treatment of intrabony defects in humans. J Periodontal Res. 2002; 37:3006.

[6]. Camargo PM, Lekovic V, Weinlaender M, Vasilic N, Madzarevic M and Kenney EB. A reentry study on the use of bovine porous bone mineral, GTR, and platelet-rich plasma in the regenerative treatment of intrabony defects in humans. Int J Periodontics Restorative Dent. 2005; 25:49-59

[7]. kazuhiro O, Hideaki Tai et al. 2005. "Platelet Rich Plasma combined with porous hydroxyapatite graft for the treatment of intrabony periodontal defect in humans. A comparative controlled clinical study". J Periodontol, 76, 890-898.

[8]. Lynch S.E. introduction in Incy S.E.genco RJ, Mark R.E. eds Tissue Engineering Applications in Maxillofacial surgery \& Periodontics Co, Inc 1999 pg 71-82

[9]. Thomas D.Pappert, Walter A. Castelli Daniel J.Chicago Effects of Platelet Derived Growth Factor on cellular response of the periodontics. An autoradiographic study on Dog.Vol.4 Pg 429

[10]. Samuel E. Lynch, Gustavo Ruiz de Castilla the effects of Short -Term Application of a combination of Platelet-derived and Insulin- Like Growth Factor on periodontal Wound Healing. J Periodontol 1991 Vol 62: 458-467.

[11]. Tolga Fikret Tozum : Platelet rich plasma : A promising Innovation in dentistry: JCan Dent Assoc 2003 Vol: 69, No.10 664

[12]. Dori F, Kovacs V, Arweiler NB, Huszar T, Gera I, Nikolidakis D and Sculean A. Effect of platelet-rich plasma on the healing of intrabony defects treated with an anorganic bovine bone mineral: a pilot study. J Periodontol. 2009; 80:1599-605.

[13]. Sukumar S, Drizhal I, Bukac J, Paulusova V and Pilathadka S. Surgical treatment of periodontal intrabony defects with calcium sulphate in combination with beta tricalcium phos phate--a 12-month retrospective clinical evaluation. Acta Medica (Hradec Kralove). 2010; 53:229-34. 
[14]. Hanna R, Trejo PM and Weltman RL. Treatment of intrabony defects with bovine-derived xenograft alone and in combination with platelet-rich plasma: a randomized clinical trial. J Periodontol. 2004;75:1668-77.

[15]. Yilmaz S, Kabadayi C, Ipci SD, Cakar G and Kuru B. Treatment of intrabony periodontal defects with platelet-rich plasma versus platelet-poor plasma combined with a bovine-derived xenograft: a controlled clinical trial. J Periodontol. 2011; 82:837-44.

[16]. Saini N, Sikri P and Gupta H. Evaluation of the relative efficacy of autologous platelet-rich plasma in combination with betatricalcium phosphate alloplast versus an alloplast alone in the treatment of human periodontal infrabony defects: a clinical and radiological study. Indian J Dent Res. 2011;22:107-15

[17]. Kaushick BT, Jayakumar ND, Padmalatha O and Varghese S. Treatment of human periodontal infrabony defects with hydroxyapatite + beta tricalcium phosphate bone graft alone and in combination with platelet rich plasma: a randomized clinical trial. Indian J Dent Res. 2011; 22:505-

[18]. Shreya Shetty ${ }^{*}$ and Aditi Bose A clinical and radiographic evaluation of the management of periodontal osseous defects with alloplast and platelet rich plasma Journal of Regenerative Medicine \& Tissue Engineering 2.11

[19]. ISSN 2050-1218

[20]. Sakshi Ahuja ${ }^{1}$, Vidya Dodwad ${ }^{2}$, Bhavna Jha Kukreja ${ }^{2}$, Pankaj Kukreja ${ }^{3}$, Praful Mehra ${ }^{4}$ A comparative evaluation of platelet-rich plasma in combination with demineralized freeze-dried bone allograft and DFDBA alone in the treatment of periodontal intrabony defects: A clinicoradiographic study.Journal of Indian Society of Periodontology, Vol. 18, No. 5, September-October, 2014, pp. $618-623$ 